\title{
Erythropoietin stimulating agents in the management of anemia of chronic kidney disease
}

\author{
Amir Hayat \\ Dhiren Haria \\ Moro O Salifu \\ Division of Nephrology, Department \\ of Medicine, SUNY Downstate \\ Medical Center, Brooklyn, NY, USA
}

\begin{abstract}
Anemia is a very common clinical problem in patients with chronic kidney disease (CKD) and is associated with increased morbidity and mortality in these patients. Erythropoietin is a hormone synthesized in the kidney responsible for red blood cell maturation in the bone marrow. It is deficient in the majority of patients with advanced kidney disease thereby predisposing to anemia. Since the approval of recombinant human erythropoietin (epoetin alfa) by the US FDA in 1989, epoetin alfa and similar agents now collectively known as erythropoietin stimulating agents (ESA) have become the standard of care for the treatment of the erythropoietin-deficient anemia that occurs in most patients with CKD. In this review, we have outlined the considerations that need to be taken into account when prescribing ESA for the treatment of anemia in CKD.
\end{abstract}

Keywords: erythropoietin stimulating agents, chronic kidney disease, anemia

\section{Introduction}

The National Kidney Foundation of The United States of America (Levey et al 2003) defines chronic kidney disease (CKD) as (a) evidence of kidney damage based on abnormal urinalysis results (eg, proteinuria, hematuria) or structural abnormalities observed on ultrasound images or (b) an absolute glomerular filtration rate (GFR) of less than $60 \mathrm{~mL} / \mathrm{min}$ for 3 or more months. Based on this definition, guidelines are developed that classify the progression of renal disease into 5 stages, from kidney disease with a preserved GFR to end-stage kidney failure. In Stage 1, there is evidence of kidney damage but GFR is preserved ( $>90 \mathrm{~mL} / \mathrm{min}$ ). Stage 2 is mild kidney damage with GFR $60-90 \mathrm{~mL} / \mathrm{min}$; Stage 3 is moderate kidney damage with GFR $30-59 \mathrm{~mL} / \mathrm{min}$; Stage 4 is severe kidney damage with GFR $15-29 \mathrm{~mL} / \mathrm{min}$ while Stage 5 is end stage renal disease (ESRD) with GFR $<15 \mathrm{~mL} / \mathrm{min}$. Patients in Stage 5 are often treated with dialysis or kidney transplantation.

CKD is a worldwide public health problem. In the US alone, over 30 million people are afflicted with CKD and the number of patients eventually reaching ESRD is projected to rapidly increase from 450,000 in 2003 to 661,330 by the year 2010 (US RDS 2005). This high prevalence of CKD, the attendant need for anemia treatment with ESA, and the high costs associated with anemia treatment in CKD calls for a more structured approach on the use of these agents.

Anemia defined as hemoglobin concentration below $13.0 \mathrm{~g} / \mathrm{dL}$ for adult males and post-menopausal women, and a hemoglobin below $12.0 \mathrm{~g} / \mathrm{dL}$ for pre-menopausal women (World Health Organization 1968), is a major co-morbidity of CKD and is common in all stages but becomes more pronounced at the latter stages of kidney failure (McClellan et al 2004). Erythropoietin deficiency is the most significant cause of anemia in CKD and has been demonstrated to occur at each stage of kidney failure. Because the kidney is the sole source of erythropoietin (EPO) synthesis in adults, reduction in kidney mass as occurs in progressive $\mathrm{CKD}$ often results in impairment of 
EPO production, resulting in anemia. Except for polycystic kidney disease, in which EPO production is preserved due to the fact that the epithelial cells of the cysts produce large amounts of EPO, other causes of kidney failure, including diabetes, hypertension, glomerulonephritis, are universally associated with anemia, which worsens as kidney failure progresses. Although recombinant human erythropoietin is a substitute for the deficiency observed in CKD, therapy of anemia often involves many other issues detailed below that need to be considered in order to effectively correct anemia, reduce costs and minimize side effects.

\section{Impact of anemia on health}

Anemia is a contributing factor in many of the symptoms associated with reduced kidney function. These include fatigue, depression, reduced exercise tolerance and dyspnea. In addition, anemia has direct adverse cardiovascular disease (CVD) consequences (McFarlane et al 2006), such as left ventricular hypertrophy ( $\mathrm{LVH})$ and left ventricular systolic dysfunction, coronary artery disease, and stroke (McLellan et al 2002; Jurkowitz et al 2003). As a result, patients with anemia due to CKD are at increased risk of hospitalization, increased hospital length of stay, reduced quality of life and mortality (Ma et al 1999; Collins et al 2000).

\section{Types of erythropoietin stimulating agents (ESA) used in treating anemia in CKD}

The first recombinant human erythropoietin (Epogen ${ }^{\circledR}$ [epoetin alfa], Amgen) in 1989 (Eschbach et al 1989) significantly improved the clinical management of anemia of CKD, primarily in dialysis patients. By $2005,99 \%$ of in-center hemodialysis patients received ESA treatment for their anemia, as outlined in Table 1. ESA therapy is the largest single Medicare drug expenditure in the US, totaling US $\$ 1.8$ billion in 2004 and comprised 11\% of all Medicare ESRD costs (Mae et al 2007). In the US, epoetin alfa is also marketed as Procrit $^{\circledR}$ (Ortho Biotech) primarily in pre-ESRD patients. In Europe epoetin alfa (Eprex ${ }^{\circledR}$, Ortho Biotech) and epoetin beta (Neorecormon $^{\circledR}$, Roche), different only in glycosylation were approved for the treatment of anemia of CKD. The above ESA are first generation and typically have shorter half-lives, requiring frequent dosing 1 to 3 times weekly. Darbopoetin alfa (Aranesp ${ }^{\circledR}$, Amgen), considered 2nd generation ESA, has been modified from EPO to give it a longer half life and typically can be administered once weekly or once every two weeks. Recently, a third generation ESA that is modified from
EPO by insertion of a large pegylation chain to make it longer acting, called continuous EPO receptor activator $\left(\mathrm{Cera}^{\circledR}\right)$ has been approved for marketing as Mircera ${ }^{\circledR}$ (Roche). The action of Mircera ${ }^{\circledR}$ on the EPO receptor is different from the other ESA, giving it a much longer half life and typically can be administered once every two weeks or once every month (Macdougall 2008). Irrespective of the type of ESA used, the common goal is to correct anemia to target levels. The choice of ESA depends on geographic, economics, industry pressure, and local policies.

\section{Considerations in the use of ESA in CKD Etiology of anemia \\ Erythropoietin deficiency}

As shown in Table 2, EPO deficiency is by far the leading cause of anemia in patients with CKD. The kidney is the primary site of erythropoietin production in adults (Ratcliffe et al 1995). Studies utilizing transgenic mice suggest that a population of interstitial fibroblasts (also known as the type I interstitial cell) are the major source of renal erythropoietin synthesis (Maxwell et al 1993). Deficiency of EPO, as occurs in patients with CKD, retards maturation of red blood cells from progenitor cells into normoblasts and reticulocytes. Furthermore, deficiency of EPO decreases the survival of these immature red blood cells, a process known as neocytolysis, thereby resulting in anemia (Righetti et al 2004). Therefore EPO supplementation is indicated in patients with CKD who manifest anemia. In the absence of other causes, anemia due to EPO deficiency is often normocytic and normochromic, implying a reduction in number but not quality of these cells. Because of the strong

Table I Currently available erythropoietin stimulating agents

\begin{tabular}{|c|c|c|}
\hline Agent & Biotech company & Main market \\
\hline \multicolumn{3}{|l|}{ First generation ESA } \\
\hline Epoetin alfa (Epogen) & Amgen & US \\
\hline Epoetin alfa (Procrit) & Ortho Biotech & US \\
\hline Epoetin alfa (Eprex) & Janssen-Ortho & $\begin{array}{l}\text { Worldwide } \\
\text { except US }\end{array}$ \\
\hline $\begin{array}{l}\text { Epoetin beta } \\
\text { (NeoRecormon) }\end{array}$ & Roche & Europe \\
\hline \multicolumn{3}{|l|}{ Second generation ESA } \\
\hline Darbopoetin (Aranesp) & Amgen & $\begin{array}{l}\text { North America, } \\
\text { Europe, Canada }\end{array}$ \\
\hline \multicolumn{3}{|l|}{ Third generation ESA } \\
\hline $\begin{array}{l}\text { Continuous } \\
\text { erythropoietin receptor } \\
\text { activator (Mircera) }\end{array}$ & Roche & Europe \\
\hline
\end{tabular}


Table 2 Considerations in the use of erythropoietin stimulating agents in chronic kidney disease

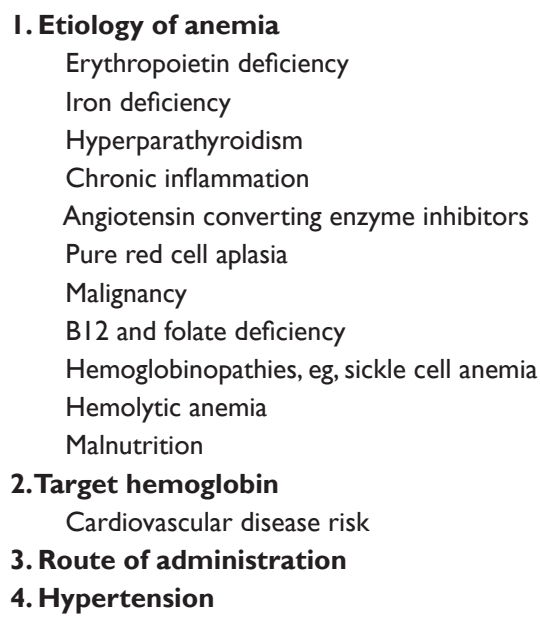

association between CKD and EPO deficiency, it is often not necessary to measure serum erythropoietin levels prior to treating anemia in these patients. In the presence of other causes of anemia detailed below, therapy with recombinant erythropoietin alone may not necessarily correct anemia, a process known as erythropoietin resistance.

\section{Iron deficiency}

Iron deficiency is prevalent in CKD and dialysis patients. Iron deficiency may result from nutritional deficiency as well as blood losses from occult gastrointestinal hemorrhage, menstruation, and blood draws. Blood draws are particularly significant in hemodialysis patients in whom up to $2 \mathrm{~g}$ of iron can be lost per year (KDOQI 2006). Furthermore, EPO therapy is associated with increased iron utilization, further leading to iron deficiency. Thus, iron deficiency will develop in virtually all dialysis patients receiving EPO unless supplemental iron therapy is given intravenously. In fact aggressive diagnosis and therapy of iron deficiency is the singular cost effective strategy in reducing ESA requirements in CKD patients (Fishbane et al 1995).

Evaluation of iron stores should include red blood cell indices, reticulocyte count, serum iron, total iron binding capacity, percentage transferrin saturation, serum ferritin, and testing for occult blood in stool. Other less commonly used indices of iron deficiency include reticulocyte $\mathrm{Hb}$ Content $(\mathrm{CHr})<29 \mathrm{pg}$ and percentage of hypochromic red cells $(\mathrm{PHRC})<10 \%$. In patients with $\mathrm{CKD}$, absolute iron deficiency is defined as serum ferritin $<100$ ( $<200$ for hemodialysis patients) $\mathrm{ng} / \mathrm{mL}$ and or a transferrin saturation $<20 \%$. In contrast, some patients often manifest 'functional' or 'relative iron deficiency' in which demand for iron in erythropoiesis exceeds supply with the administration of ESA. Iron stores are adequate in functional iron deficiency; however, there is an inability to adequately mobilize it from the reticuloendothelial system (RES) to support erythropoiesis. Patients with functional iron deficiency anemia often respond to intravenous iron (Coyne et al 2007) as opposed to a condition termed reticuloendothelial blockade, an extreme case of functional iron deficiency usually occurring in the setting of acute or chronic inflammation/infection. This often correlates with a high CRP level and/or a high erythrocyte sedimentation rate. Typically the serum ferritin level is $>200 \mathrm{ng} / \mathrm{mL}$ but the transferrin saturation $<20 \%$ in both conditions. Hepcidin, a peptide produced by the liver, inhibits intestinal iron absorption and the release of iron from the RES to circulating transferrin. Serum levels of hepcidin are increased in the setting of inflammation/infection, and help explain the phenomenon of RES blockade in which stored iron is not released to circulating transferrin, resulting in a high serum ferritin and low transferring saturation level (Wish 2006). Both functional iron deficiency and reticuloendothelial blockade may lead to EPO resistance (Tonelli et al 2001; Brugnara 2003). Serum ferritin and transferrin saturation should be measured before initiating ESA therapy and every 1-3 months during therapy. Patients with stable hemoglobin in the target range who are receiving a stable dose of an ESA should have their hemoglobin checked monthly and any evidence of iron deficiency should be treated with parenteral iron as this route of administration is more effective.

\section{Secondary hyperparathyroidism}

Secondary hyperparathyroidism parallels CKD and worsens with its severity. This is due primarily to deficiency in 1-25 vitD3 and elevated serum inorganic phosphate level both of which stimulate parathyroid hyperplasia and increased parathyroid hormone synthesis, a process called secondary hyperparathyroidism. Secondary hyperparathyroidism increases bone turnover, leading to development of bone cysts and marrow fibrosis, impairing bone marrow function and subsequently anemia (Barany and Muller 2007). Secondary hyperparathyroidism, if uncontrolled, can lead to erythropoietin resistance. Therefore secondary hyperparathyroidism must be routinely checked and aggressively treated in CKD patients.

\section{Chronic inflammation}

Chronic inflammation is an important cause of EPO resistance in CKD patients. Chronic inflammation resulting from 
rheumatologic diseases (such as arthritis, lupus), chronic infections (such as hepatitis $\mathrm{C}$, soft tissue and bone infections), and failed renal transplants is associated with increased circulating levels of cytokines including CRP, tumor necrosis factor, interferon gama, and interleukins (Tsirpanlis et al 2004), which impair the erythropoietic response to various stimuli. As CRP has emerged as the most quantifiable marker of inflammation, it should be routinely investigated when erythropoietin resistance is suspected and no other cause can be identified. In general, levels of CRP $>10 \mathrm{mg} / \mathrm{L}$ (Ortega et al 2002), suggest significant inflammation. Occasionally doubling the erythropoietin dose may overcome the resistance. If no improvement is achieved with doubling the dose, further increments are not likely to yield significant results. In such instances, the erythropoietin doses should not be stopped as this may precipitate neocytolysis, resulting in precipitous decline in red cell count.

Diagnosis of the underlying cause of inflammation is key in order to eliminate the cause of elevated CRP. If infection is diagnosed specific antibiotic therapy or surgical intervention can improve EPO resistance. Similarly, detection and treatment of recurrent vasculitis or malignancy may also have a high impact. If no cause of high CRP is detected a clotted arteriovenous graft should be removed, when appropriate. On rare occasions, if the screen for sepsis is negative and the patient is believed to have a non-infective inflammatory condition, administration of steroids might be considered (Wanner et al 2007). Alternatively, such patients should be maintained on regular doses of erythropoietin and periodic red blood cell transfusion.

\section{Pure red cell aplasia}

Although rare, administration of ESA may result in formation of anti-erythropoietin antibodies, thereby leading to pure red cell aplasia and erythropoietin resistance (Pollocke et al 2008). In patients in whom ESA doses have been maximized without effect and no other causes can be identified, serum anti-erythropoietin levels and bone marrow biopsy should be performed. If confirmed, erythropoietin administration should be ceased and the patient treated with periodic blood transfusions.

\section{Angiotensin converting enzyme inhibitors and receptor blockers}

Angiotensin converting enzyme inhibitors (ACEI) and angiotensin receptor blockers (ARB) can contribute to anemia in CKD patients. The observation that ACEI and ARB are preferred drugs in treating post-transplant erythrocytosis support the impact of these agents on anemia. Furthermore, evidence suggests that dialysis patients treated with ACEI and ARB have slightly lower hematocrits than those not on these agents. A circulating natural inhibitor of bone marrow (N-acetyl-seryl-aspartyl-lysyl-proline (AcSDKP) has been implicated in the pathogenesis of ACEI-induced anemia. The ACE enzyme is responsible for the degradation of AcSDKP, thus blockade of ACE with ACEI leads to increased circulating levels of AcSDKP and therefore bone marrow inhibition (Le Meur et al 2001). Secondly, erythroid progenitor cell lines can be stimulated via angiotensin type II receptors therefore blockade of these receptors by ARB may inhibit differentiation of erythroid cell lines (Mohanram et al 2008). In most instances increase in dose of erythropoietin may overcome the resistance induced by ACEI or ARB. If necessary, modest reduction of doses of ACEI or ARB may be needed in CKD patients with unexplained anemia. Because of the overall cardiovascular benefit, in the absence of serious complications, complete cessation of ACEI and ARB for the purpose of treating anemia is not indicated.

\section{Hemolytic anemia}

Hemolytic anemia is associated with various conditions in CKD patients including systemic lupus erythematosus and microangiopathic hemolytic anemia. Tests of hemolysis such as total bilirubin, lactate dehyrogenase, urine hemosiderin, urine hemoglobin, haptoglobin, and a peripheral smear should be performed to diagnose the presence of hemolysis. By far, haptoglobin is the most specific of these tests and a depressed value indicates significant hemolysis. Confirmation of immune hemolytic anemia by the above tests requires complete hematologic evaluation and possible immunosuppressive therapy that is beyond the scope of this article.

\section{Other causes of anemia}

As shown in Table 2, other rare causes of anemia in CKD patients include folate and B12 deficiency, hemoglobinopathies, malignancy, and malnutrition. These causes should be routinely investigated and treated.

\section{Target hemoglobin levels}

Although the National Kidney Foundation of USA Kidney and Dialysis Outcomes and Quality Initiative (K/DOQI) guidelines on anemia suggested a hemoglobin target range of 11-12 $\mathrm{g} / \mathrm{dL}$ rather than full normalization, investigators have questioned whether normalizing hemoglobin may lead to additional benefits (Foley 2006). Two large recent randomized controlled trials; Correction of Hemoglobin 
and Outcomes in Renal Insufficiency (CHOIR) (Singh et al 2006) and Cardiovascular Risk Reduction by Early Anemia Treatment with Epoetin (CREATE) (Drueke et al 2006) demonstrated increased cardiovascular events and progression to dialysis in patients assigned to the highest hemoglobin targets $(>13.0 \mathrm{~g} / \mathrm{dL})$, compared with $<12 \mathrm{~g} / \mathrm{dL}$. This prompted new guidelines published by KDOQI in August 2007 recommending a hemoglobin target range of $11-12 \mathrm{~g} / \mathrm{dL}$ for patients with CKD (KDOQI 2007). Subsequently the US Food and Drug Administration (FDA) issued an advisory (Public Health Advisory; Information for Healthcare Professionals) recommending hemoglobin within the range of 10-12 g/dL, with more frequent hemoglobin monitoring (twice weekly), so that early adjustment to ESA therapy may be instituted to avert significant over treatment. Furthermore, ESA therapy with target hemoglobin above $13.0 \mathrm{~g} / \mathrm{dL}$ has been associated with increased progression of many different types of cancers. The FDA has warned that ESA should be administered only to avoid red blood cell transfusions in cancer patients as they do not improve the outcome of cancer treatment and do not alleviate fatigue or increase energy in these patients.

Whether the increase risk of cardiovascular events, progression to dialysis and cancer progression is due to higher doses of ESA required to achieve those targets, or is due to the increase in hemoglobin per se or a combination of both factors is still a subject of debate. Nonetheless, these potential complications are important considerations when prescribing ESA. In patients with history of cardiovascular disease or cancer, strict adherence to the recommended targets should be the goal.

\section{Route of administration}

The route of administration should be taken into account when administering ESA in CKD. Evidence suggests that the subcutaneous route usually results in less frequent injections, reduces ESA requirement and is cost effective than the intravenous route (Tonelli et al 2003). Despite this fact, many dialysis patients and perhaps nephrology professional staff use the intravenous route since ESA can be conveniently administered during the process of dialysis. For instance, in the USA, most patients on dialysis receive ESA therapy intravenously. In peritoneal dialysis patients and in the non-dialysis CKD population the choice is however limited to subcutaneous administration since patients often are instructed to inject themselves at home without supervision using the least invasive method, which is subcutaneous. In a few patients who do not exhibit adequate response to intravenous ESA administration despite dose adjustment, consideration should be given to switching to the subcutaneous route. When this switch is considered, the subcutaneous dose should be reduced by $30 \%$ to avoid the possibility of a sudden rise in hemoglobin above target.

\section{Uncontrolled hypertension}

Administration of ESA may be associated with exacerbation of hypertension in about $5 \%$ of patients that will require dose adjustment or switching to a different ESA. In the era of new and effective antihypertensive therapy, rarely does full cessation of ESA become necessary. The mechanism of ESA induced hypertension is thought to be related to stimulation of the vascular endothelium by ESA resulting in increased circulating levels of endothelin. Furthermore the increase in hemoglobin associated with ESA therapy may increase blood viscosity resulting in vasospasm. As such routine monitoring of blood pressure is essential in patients treated with ESA (Public Health Advisory; Information for Healthcare Professionals).

\section{Conclusion}

Treatment of anemia with ESA in patients with CKD is indicated to improve quality of life and decrease morbidity and mortality. A variety of issues raised in this article need to be taken into account when prescribing ESA to treat anemia in CKD. Furthermore as emerging data suggests that ESA therapy may not be that benign, it is imperative to adhere to strict guidelines and keep hemoglobin levels on the recommended targets ranges. Such action can only be achieved by frequent monitoring of the patient to improve overall outcomes.

\section{References}

Barany H-J, Muller. 2007. Maintaining control over haemoglobin levels: optimizing the management of anaemia in chronic kidney disease. Nephrol Dial Transplant, 22(Supp1 4):iv10-iv18.

Brugnara C. 2003. Iron deficiency and erythropoiesis: new diagnostic approaches. Clin Chem, 49:1573-8.

Collins AJ, Ma JZ, Ebben J. 2000. Impact of hematocrit on morbidity and mortality. Semin Nephrol, 20:345.

Coyne, DW, Kapoian, T, Suki, W, et al. 2007. Ferric gluconate is highly efficacious in anemic hemodialysis patients with high serum ferritin and low transferrin saturation: Results of the dialysis patients' response to IV iron with elevated ferritin (DRIVE) study. J Am Soc Nephrol, 18:975.

Drueke TB, Locatelli F, Clyne N, et al. 2006. Normalization of hemoglobin level in patients with chronic kidney disease and anemia. $N$ Engl J Med, 355:2071-84.

Eschbach JW, Kelly MR, Haley NR, et al. 1989. Treatment of the anemia of progressive renal failure with recombinant human erythropoietin. N Engl J Med, 20:158-63.

Fishbane S, Frei GL, Maesaka J. 1995. Reduction in recombinant human erythropoietin doses by the use of chronic intravenous iron supplementation. Am J Kidney Dis, 26:41-6. 
Foley RN. 2006. Do we know the correct hemoglobin target for anemic patients with chronic kidney disease? Clin J Am Soc Nephrol, 1:678-84.

Information for Healthcare Professionals: Erythropoiesis Stimulating Agents (ESA) [Aranesp (darbepoetin), Epogen (epoetin alfa), and Procrit (epoetin alfa) [online] Accessed July 17, 2008. URL: http://www.fda. gov/cder/drug/InfoSheets/HCP/RHE2007HCP.htm.

Jurkovitz CT, Abramson JL, Vaccarino LV, et al. 2003. Association of high serum creatinine and anemia increases the risk of coronary events: results from the prospective community-based atherosclerosis risk in communities (ARIC) study. J Am Soc Nephrol, 14:2919.

KDOQI Clinical Practice Guideline and Clinical Practice Recommendations for anemia in chronic kidney disease. 2007. Update of hemoglobin target Am J Kidney Dis, 50:471-530.

KDOQI Clinical Practice Guidelines and Clinical Practice Recommendations for Anemia in Chronic Kidney Disease. 2006. Am J Kidney Dis, 47:S11-145

Le Meur Y, Lorgeot V, Comte L, et al. 2001. Plasma levels and metabolism of AcSDKP in patients with chronic renal failure: relationship with erythropoietin requirements. Am J Kidney Dis, 38:510-7.

Levey AS, Coresh J, Balk E, et al. 2003. National Kidney Foundation Disease Outcomes Quality Initiative Clinic Practice Guidelines for Chronic Kidney Disease: Evaluation, Classification and Stratification. Ann Intern Med, 139:137-47.

Ma JZ, Ebben J, Xia, H Collins, AJ. 1999. Hematocrit level and associated mortality in hemodialysis patients. $J$ Am Soc Nephrol, 10:610.

Macdougall IC. 2008. Novel erythropoiesis-stimulating agents: a new era in anemia management. Clin J Am Soc Nephrol, 3:200-7.

Mae Thamer, Yi Zhang, James Kaufman, et al. 2007. Dialysis Facility Ownership and Epoetin Dosing in Patients Receiving Hemodialysis. JAMA, 297:1667-74.

Maxwell PH, Osmond MK, Pugh CW, et al. 1993. Identification of the renal erythropoietin-producing cells using transgenic mice. Kidney Int, 44:1149.

McClellan W, Aronoff SL, Bolton WK, et al. 2004. The prevalence of anemia in patients with chronic kidney disease. Curr Med Res Opin, 20:1501-10.

McClellan WM, Flanders WD, Langston RD, et al. 2002. Anemia and renal insufficiency are independent risk factors for death among patients with congestive heart failure admitted to community hospitals: a population-based study. J Am Soc Nephrol, 13:1928.
McFarlane SI, Salifu MO, Makaryus J, et al. 2006. Anemia and cardiovascular disease in diabetic nephropathy. Curr Diab Rep, 6:213-8.

Mohanram A, Zhang Z, Shahinfar S, et al. 2008. The effect of losartan on hemoglobin concentration and renal outcome in diabetic nephropathy of type 2 diabetes. Kidney Int, 73:630-6.

Ortega O, Rodriguez I, Gallar P, et al. 2002. Significance of high C-reactive protein levels in pre-dialysis patients. Nephrol Dial Transplant, 17:1105-9.

Pollock C, Johnson DW, Hörl WH, et al. 2008. Pure red cell aplasia induced by erythropoiesis-stimulating agents. Clin J Am Soc Nephrol, 3:193-9.

Public Health Advisory: Epoetin alfa (marketed as Procrit, Epogen), Darbepoetin alfa (marketed as Aranesp) [online] Accessed July 17, 2008.URL:http://www.fda.gov/cder/drug/advisory/RHE.htm.

Ratcliffe, PJ, Ebert, BL, Ferguson, DJ, et al. 1995. Regulation of the erythropoietin gene. Nephrol Dial Transplant, 10(Suppl 2):18.

Righetti M, Ferrario GM, Milani S, et al. 2004. A single centre study about the effects of HFR on anemia. G Ital Nefrol, 21(Suppl 30):S168-71.

Singh AK, Szczech L, Tang KL, et al. 2006. Correction of anemia with epoetin alfa in chronic kidney disease. N Engl J Med, 355:2085-98.

Tonelli M, Blake PG, Muirhead N. 2001. Predictors of erythropoietin responsiveness in chronic hemodialysis patients. ASAIO J, 47:82-5.

Tonelli M, Winkelmayer WC, Jindal KK, et al. 2003. The cost-effectiveness of maintaining higher hemoglobin targets with erythropoietin in hemodialysis patients. Kidney Int, 64:295-304.

Tsirpanlis G, Bagos P, Ioannou D, et al. 2004. Exploring inflammation in hemodialysis patients: persistent and superimposed inflammation. A longitudinal study. Kidney Blood Press Res, 27:63-70.

US Renal Data System, USRDS 2005 Annual Data Report: Atlas of End-Stage Renal Disease in the United States, National Institutes of Health, National Institute of Diabetes and Digestive and Kidney Diseases, Bethesda, MD

Wanner C, Richardson D, Fouque D, et al. 2007. OPTA - Influence of inflammation/infection on anaemia therapy in haemodialysis patients. Nephrol Dial Transplant, 22(Suppl 3):iii7-iii12; doi:10.1093/ ndt/gfm015.

Wish JB. 2006. Assessing iron status: beyond serum ferritin and transferrin saturation. Clin J Am Soc Nephrol, 1: S4-S8.

World Health Organization. Nutritional Anaemias. 1968. Report of a WHO Scientific Group. Geneva, Switzerland: World Health Organization. 